Article type : Regular Paper

\title{
Principal mode of Syndecan-4 mechanotransduction for the endothelial glycocalyx is a scissor-like dimer motion
}

\author{
Xi Zhuo Jiang, Kai H. Luo, Yiannis Ventikos \\ Department of Mechanical Engineering, University College London, Torrington Place,
} London WClE 7JE, UK

Short title: Scissor motion for mechanotransduction

*Corresponding Authors:

Emails: y.ventikos@ucl.ac.uk (Yiannis Ventikos); k.luo@ucl.ac.uk (Kai H. Luo)

Postal Address: Department of Mechanical Engineering, University College London, Torrington Place, London WC1E 7JE, UK

\section{Abstract}

\section{Aim:}

Endothelial glycocalyx plays a pivotal role in a plethora of diseases, like cardiovascular and renal diseases. One hallmark function of the endothelial glycocalyx as a mechanotransducer which transmits mechanical signals into cytoplasm has been documented for decades.

This article has been accepted for publication and undergone full peer review but has not been through the copyediting, typesetting, pagination and proofreading process, which may lead to differences between this version and the Version of Record. Please cite this article as doi: 10.1111/apha.13376

This article is protected by copyright. All rights reserved. 
However, the basic question - how the glycocalyx transmits the flow shear stress - is unanswered so far. Our aim is to shed light on the fundamental mode of signal transmission from flow to the endothelial cytoskeleton.

\section{Methods:}

We conduct a series of large-scale molecular dynamics computational experiments to investigate the dynamics of glycocalyx under varying conditions (changing blood flow velocities and shedding of glycocalyx sugar chains).

\section{Results:}

We have identified that the main pathway of signal transmission in this system manifests as a scissors-like motion of the Syndecan-4 core protein. Results have suggested that the force transmitted into the cytoskeleton with an order of $10 \sim 100 \mathrm{pN}$, and the main function of sugar chains of a glycocalyx element is to protect the core proteins from severe conformational changes thereby maintaining the functionality of the EG.

\section{Conclusion:}

This research provides a reconciling explanation for a longstanding debate about the force transmission threshold based on our findings. A new explanation has also been provided to relate the role of the EG as a mechanotransducer to its function as a microvascular barrier: the EG regulates the mechanotransduction by altering the median value and variation range of the scissor angle, and the EG governs the microvascular barrier via controlling the scissor angle which will affect the intercellular cleft. 


\section{Keywords}

endothelial glycocalyx, flow shear stress, mechanotransduction, molecular dynamics simulation, Syndecan-4

\section{Introduction}

The ubiquitous endothelial glycocalyx (EG), exposed to the mechanical forces of blood flow, plays a profound role in vascular regulation ${ }^{1}$, health, diseases ${ }^{2,3}$ and therapies ${ }^{4}$. Experiments have shown that the EG is present as soon as blood flow is initiated ${ }^{5}$ and is required for proper vascular development (e.g. remodelling ${ }^{6}$ ). Vascular diseases (atherosclerosis, hypertension, stroke, renal diseases and sepsis ${ }^{7-9}$ ) are associated with a degraded EG, whereas growth and metastasis of cancer cells are associated with an overly robust ${ }^{10}$. As the abnormal activities of the EG impair the functionality of the vascular endothelium, therapeutic strategies targeting the EG are developed to intervene its activities and inhibit the development or progression of various diseases are expected to ameliorate the pathology ${ }^{4}$. For example, the disintegration of EG occurs very early in the course of sepsis and its prevention improves the survival of mice with sepsis, and pharmacologic acceleration of EG restoration can be achieved using sulodexide ${ }^{9}$.

Mechanotransduction ${ }^{11-13}$, which involves biomolecules sensing mechanical signals ${ }^{14-16}$ (flow shear stress ${ }^{17}$ being of interest in this work) and transducing these physical cues into the intracellular biomolecules, is a hallmark function of the EG. The transmitted signals can cause oxidant production, and the resulting redox signals initiate inflammation signalling 
pathways which in turn can compromise vascular health. Previous work has shown that endothelial mechanotransduction signalling pathways participate in generation of redox signals that affect inflammation status of cells ${ }^{18}$. However, to this date, the actual intermediate signals of flow-induced, shear stress-related mechanotransduction have not been elucidated, and the role of EG in this regard is unresolved ${ }^{19}$. What is known is that enzymatic degradation of the EG constituents decreases flow-induced nitric oxide (a vasoregulating agent responding to shear stress changes) production ${ }^{20}$ confirming that EG plays an important role in such signal transmission.

To investigate the functionality of EG, conducting wet-lab experiments is definitely an effective and straightforward method. As mentioned previously, the enzymatic degradation has identified the importance of EG in signal transmission. However, limitations of enzymatic degradation should also be noted. For example, proteoglycans can be found both on the apical and basal surfaces of the endothelial cells, and the disruption of the apical proteoglycan could also cause damage to the basal surface. Therefore, enzymatic degradation studies may result in biased estimates of the relative contribution of different EG layers to mechanical functions, as commented in Ref. ${ }^{21}$. Another challenge in the experimental design is to retain the intact EG: as EG is a highly dynamic and fragile structure, its components can be lost during fixation, dehydration, sectioning and staining procedures ${ }^{8}$.

This article is protected by copyright. All rights reserved. 
The application of computational/mathematical approaches to address theoretical and experimental questions in biology has significantly broadened our horizons about the EG. Just to name a few, Dabagh et al. developed a three-dimensional, multi-scale, multi-component, and viscoelastic model of focally adhered endothelial cells, and discussed the contributions of the substructures to mechanotransduction under complicated flow conditions ${ }^{22}$. Eriksson et al. performed classical-mechanical molecular dynamics simulations to study solvation properties of simple cations of biological relevance and reported that the glycocalyx oligosaccharides prefer direct contact with $\mathrm{K}^{+}$over $\mathrm{Na}^{+}$, but that the $\mathrm{Na}^{+}$contacts are longer lived ${ }^{23}$. Such computational studies are distinctive as experiments are unlikely to reveal the interactions between endothelium and its ambient environment in such detail. Computational experiments lend the possibility to circumvent the limitations in wet-lab experimental methods. Meanwhile, they also provide additional insights and details that experiments cannot offer.

Among the computational methods, molecular dynamics (MD) is a powerful tool to reveal the atomic events concealed inside the EG layer. However, how the MD method can reveal the hidden mystery behind the complex biomolecular layers is restricted by the resolution of biomolecular structures and the capacity of computational hardware as well. Before the publication of the detailed structure of a typical EG element ${ }^{24}$, early MD research can only deal with a few sugar residues with one end tethered on a fixed plane were simulated ${ }^{23}$. With the advent of the detailed structures and the implementation of supercomputers, a series of interesting phenomena regarding the EG layer have been 
discovered, and most of these findings are from our group. For example, the blood flow alters the conformation of the EG ${ }^{25-28}$; the conformational changes then affect their interactions with the surrounding ions, modifying the microvascular ion transport properties ${ }^{29-31}$. However, these studies mainly focus on atomic/molecular events occurring in the ectodomain. The mechanism for the EG implementing its transmembrane functionality (mechanotransduction) remains undeciphered. As the EG is also essential in regulating the endothelial permeability, are there any connections between roles of the EG as a mechanotransducer and a microvascular barrier? All of these unclear questions are related to a central mechanism how does the EG transmit force from the ectodomain to the cytoplasm?

To clarify this mechanism in the present research, we construct a flow/EG system with a fine resolution as shown in Figure 1A. In our system, the EG element with Syndecan-4 and HS chains published in Ref. ${ }^{24}$ is adopted, as the ubiquitously expressed Syn-4 mediates numerous cellular processes like mechanotransduction ${ }^{32}$ and plays a critical role in sensing flow direction ${ }^{33}$ and HS chains account for the large quantities (50\% 90\%) of the EG glycosaminoglycan chains ${ }^{34}$. Using direct simulation - molecular dynamics modelling, we will scrutinise the dynamics of the EG, especially the core protein (Figure 1B) which bridges the ectodomain and the cytoplasm. Based on the dynamics of the EG constituents, we will then propose the force transmission mode of the EG mechanotransduction together with the force magnitude. Furthermore, the main function of sugar chains of the EG will also be discussed. Based on the findings from this research, the functionality of the EG will be interpreted from a new perspective. 


\section{Results}

The model of EG core protein (whether rigid or soft) determines the force transmission mode from the ectodomain to the cytoplasm in the mechanotransduction process of Syn-4. In early theoretical studies, an EG core protein was usually assumed to be rigid and immobile 35,36. Under this hypothesis, bending of the EG core protein via a lever fashion could be the only possible mode via which the core protein transmits forces from the ectodomain to the cytoskeleton. However, our previous research reveals that the motions of the core protein and the attached sugar chains are unsynchronised ${ }^{25}$, suggesting that the EG element behaves in a soft-matter way. Even for the EG core protein per se, the soft-matter structural information can still be revealed by its secondary structures, as the ectodomain and the transmembrane parts of the core proteins are connected by the flexible coils (Figure 1B). To be specific, the subdomain labelled EA1/EB1 is linked with the transmembrane part via a single coil. Also, within the ectodomain, a single coil bridges subdomains EA1/EB1 and EA2/EB2. To facilitate further analysis, the structures used in the present study are introduced here. In Figure 1B, EA1, EA2, EB1 and EB2 are four subdomains of the Syn-4 ectodomain part. TA1 is the first coil of Chain A from the Syn-4 transmembrane dimer with TB1 the first coil of

Chain B. TA2 and TB2 are the middle coils of the transmembrane parts of Chain A and Chain $\mathrm{B}$, respectively. TA3 and TB3 individually represent the tail coils of the transmembrane dimer. $\mathrm{COM}_{\mathrm{T}}$ is the centre of mass of the Syn-4 transmembrane dimer.

This article is protected by copyright. All rights reserved. 
To confirm the soft matter model for the EG core protein, the dynamics of the core protein was scrutinised. Three angles $\left(\theta_{\mathrm{T}}, \theta_{\mathrm{E} 1}\right.$ and $\theta_{\mathrm{E} 2}$ in Figure $2 \mathrm{~A}$; see supplementary information for calculation details) recording relative positions of the core protein subdomains were monitored under flow (with the external force of $0.003 \mathrm{fN}$ ) and stationary conditions. $\theta_{\mathrm{T}}$ is to measure the relative position of the transmembrane part to $\mathrm{COM}_{\mathrm{T}}$, and $\theta_{\mathrm{E} 1}$ and $\theta_{\mathrm{E} 2}$ represent the relative positions of the subdomains of Syn-4 ectodomain to $\mathrm{COM}_{\mathrm{T}}$. If Syn-4 was a rigid body, the relative positions of the Syn-4 subdomains should remain constant regardless of whether there is flow or not. However, as shown in Figure 2A, significant increases in $\theta_{\mathrm{E} 1}$ and $\theta_{\mathrm{E} 2}$ can be observed when flow passes by, whereas $\theta_{\mathrm{T}}$ remains stable regardless of whether there is flow or not. The inconsistent and unsynchronised movements of the core protein subdomains can also be found in the distances between subdomains (i.e. $d_{\mathrm{A} 1}, d_{\mathrm{A} 2}, d_{\mathrm{B} 1}$, and $d_{\mathrm{B} 2}$; see supplementary information for calculation details) as shown in Figure 2B. Such unsynchronisation approves the soft-matter feature of the core protein. Indeed, the soft matter model links directly to our first important finding about the force transmission mode of the Syn-4 core protein in EG mechanotransduction which will be introduced in the following results.

The helix lengths of the Syn-4 transmembrane dimer (i.e. $d_{\mathrm{TA}}$ and $d_{\mathrm{TB}}$; see supplementary information for calculation details) were also examined as displayed in Figure 2C. It is noteworthy that the transmembrane dimer of the Syn-4 can be individually regarded as inextensible due to the insignificant changes in helix lengths between situations with and without flow. 


\section{Force transmission mode and its order of magnitude}

To explore potential motion modes in which forces are transmitted via the soft EG core protein, the motion and the conformational changes of the transmembrane Syn- 4 core protein were further examined via three angles (Figure 3; see supplementary information for calculation details): $\theta_{\mathrm{AB}}$ (Figure $3 \mathrm{~A}$ ) to quantify the relevant position of the two chains of the dimer; and $\theta_{\mathrm{A}}$ and $\theta_{\mathrm{B}}$ (Figure $3 \mathrm{~B}$ ) to measure deformation of each chain. In Figure 3a, the significant variations of the angle $\theta_{\mathrm{AB}}$ in the flow case $(p<0.001)$ reveals a "scissor-like" motion of the dimer chains when the EG is exposed to flow. Meanwhile, the declining median value (Figure $3 \mathrm{~B}$ ) of $\theta_{\mathrm{AB}}$ from $55.6^{\circ}$ in the control case to $52.5^{\circ}$ in the flow case indicates that the blood flow can cause the closure of the transmembrane dimer chains. The flow also stretches Chain A with the median value of $\theta_{\mathrm{A}}$ increasing from $158.6^{\circ}$ in the control case to $161.5^{\circ}$, but exerts insignificant effects on the bending of Chain B. Since both the scissor motion of the two chains and the deformation of Chain A can contribute to the changes of $\theta_{\mathrm{AB}}$, we then postulate that force is mainly transmitted by this particular scissor motion of the dimer, alongside the stretching of one chain of transmembrane dimer. Furthermore, a 2D geometric derivation (see supplementary information) predicts that the scissor motion of the two dimer chains accounts for $53 \%$ of the change in $\theta_{\mathrm{AB}}$.

To test our postulation of the force transmission mode, principal component analysis (PCA) ${ }^{37}$ was conducted to thoroughly assess the dynamics of the transmembrane Syn- 4 core protein. PCA results in Figure 3C suggest that the scissor motion of the two chains is the first principle motion mode of the transmembrane protein, followed by two bending modes of the 
dimer in two planes (animations of the three modes can be found in Movies 1-3, respectively). These three modes constitute $97 \%$ of the transmembrane dimer motion, which corroborates our postulation that the forces are transmitted via the scissor motion and the bending of the EG transmembrane dimer. It is also worth noting that scissor motion of the transmembrane dimer occupies about $55 \%$ of all the XOZ plane modes, which validates our estimation of the $53 \%$ contribution rate to $\theta_{\mathrm{AB}}$ change.

The core proteins of the EG are usually linked with actin filaments of the cytoskeleton 21,38. The angle changes of the transmembrane proteins under the flow shear stress will result in deformations of the filaments or even rupture of the linkage between the protein and the cytoskeleton. To gain additional insight regarding the order of magnitude of the force transmitted into the cytoplasm, a steered molecular dynamics (SMD) simulation was conducted. The basic idea behind a SMD simulation is to apply an external force to one or more atoms and explore biological processes under this force, like those explored using atomic force microscopy ${ }^{39,40}$. In our SMD simulation, a constant velocity of $0.0018 \mathrm{~m} \cdot \mathrm{s}^{-1}$ has been imposed on the ectodomain end of one chain of the dimer (inner panel of Figure 3D); the angle $\left(\theta_{\mathrm{AB}}\right)$ is expected to change from $55.6^{\circ}$ to $52.5^{\circ}$ in $30 \mathrm{~ns}$. To maintain the constant velocity, the external force changes throughout the 30-ns simulation. The probability density distribution of the imposed force is drawn in Figure 3D. The probability density function (PDF) of the force (Figure 3D) indicates that forces with an order of magnitude of 10 100 pN are required for presumed angle change. Considering the rigidity of the $\alpha$-helix structure of each transmembrane dimer chain (As displayed in Figure 2C, the changes in lengths of the 
helices are negligible.), a lever principle further suggests that the force transmitted to the cytoplasm end of the dimer exhibits an order of magnitude of $10 \sim 100 \mathrm{pN}$. This order of magnitude is in good agreement with atomic force microscopy results in other biomolecular studies, such as the protein depletion ${ }^{41}$, RNA stretching ${ }^{42}$, and protein conformation changing $43-45$

\section{Flow and sugar chains influence the Syn-4 mechanotransduction}

We then explored potential influence factors associated with the mechanotransduction the blood velocity and the shedding of sugar chains which are two critical factors related to cardiovascular diseases ${ }^{21,46}$. We decreased the external force from $0.003 \mathrm{fN}$ to $0.002 \mathrm{fN}$ and $0.001 \mathrm{fN}$ in the flow case respectively, and compared the angle distributions of the transmembrane dimer chains under these situations as shown in Figure 4A. The decrease in the median value of $\theta_{\mathrm{AB}}$ with the ascending external forces indicates that the strong impulse by the external force favours the closure of the scissor motion. Meanwhile, the impulse by the external force intensifies the scissor motion, as the variation ranges of $\theta_{\mathrm{AB}}$ are amplified as the external force increases.

To unveil the functionality of the sugar chains, two scenarios with shedding sugar chains were constructed, as shown in Figure 4B. By removing three sugar chains (marked in red in case I) from the central EG element, 15 sugar chains are retained in case II; case III only contains 9 sugar chains by removing 6 sugar chains (marked in blue in case II) from the surrounding two EG elements based on case II. As illustrated in Figure 4C, the decline in the 
median value of $\theta_{\mathrm{AB}}$ suggests that one major function of the sugar chains is to resist the flow and to prevent severe conformational changes of the transmembrane dimer. The decrease in the variation range of $\theta_{\mathrm{AB}}$ after the removal of the central sugar chains (cases I and II) indicates that the removed sugar chains from the central EG element could intensify the scissor motion if they were preserved. By contrast, the increase in the variation range of $\theta_{\mathrm{AB}}$ between the two shedding sugar chains situations (cases II and III) reveals that the surrounding sugar chains impede the scissor motion of the central EG element by confining the movement of the central sugar chains connected to the core protein (see Figure S2 in the supplementary information).

\section{Discussion}

In this research, we use large-scale molecular dynamics simulations to investigate the force transmission mode of Syn-4 mechanotransduction for the EG. Based on the soft-matter features of the EG structures, we have identified that the Syn-4 core protein transmits force from the ectodomain to the cytoplasm via a scissor-like motion as well as the bending of its transmembrane dimer. The force transmitted to the cytoskeleton manifests an order of magnitude of $10 \sim 100 \mathrm{pN}$. The role of the attached sugar chains is to prevent the severe conformational changes of the core protein, thereby maintaining the functionality of the EG. It is important to put these findings in context, by discussing how they fit into or elucidate the existing literature. The MD research provides a new angle of view to retrospect classic topics in the research of EG mechanotransduction. 


\section{EG recovery time from a molecular perspective}

Previous experiments observed a time constant for EG dimensional recovery after compression by circulating cells, and the time constant was successfully explained by the rigid body model ${ }^{35}$. The existence of such a time constant can also be interpreted based on our MD results: the circulating cells alter the momentum of surrounding water molecules, and result in conformational changes of sugar chains when the momentum alteration propagates to the EG sugar chains. The momentum alteration in EG sugar chains can finally be restored by their momentum exchanges with the connected core proteins. Due to the weak correlation of motions between the sugar chains and the core protein, the momentum changes cannot be compensated immediately. It therefore can be expected a time constant for the recovery of EG conformation, which is the time constant of EG dimensional changes in the experimental observation. To determine the time constant from the atomic scale results, further MD simulations are required.

\section{Mechanotransduction threshold}

The soft matter model is also powerful in resolving some complicated and debatable issues. The question of whether glycocalyx transmits force via the core proteins has long been arguable. Weinbaum et al. ${ }^{35}$ assumed the EG element as a rigid body and predicted that a torque is generated on the cell membrane when flow passes through the EG, and the torque then results in forces, thereby deforming the cytoskeleton. In accordance to this theory, the cytoskeletal deformation would be inhibited only when all the sugar chains of the glycocalyx were removed. However, experiments ${ }^{36}$ suggested that there is a threshold for the torque: $30 \%$ 
$\sim 45 \%$ reduction of the EG sugar chains can inhibit cytoskeletal reorganisation. This threshold would be difficult to explain if the EG elements were assumed as rigid body, so experimental researchers postulated that transmission of forces might not occur through the rigid EG core protein. We argue that this discrepancy can be reconciled by the soft matter model proposed in this study. The ecto- and transmembrane subdomains of the core protein are physically connected by flexible coils. The flexibility of the linkage, on the other hand, can mediate forces from the ectodomain, which means forces can only be partially transmitted into the cytoskeleton. The mediation of the linkage explains the threshold discovered by the experiments. According to our in silico experiments, only by removing $16.7 \%$ of the sugar chains ( 3 out of 18 sugar chains are removed in case II), the mechanotransduction in terms of scissor angular variation ranges has been dramatically reduced by $55 \%$, which validates the existence of the proposed threshold. It is noteworthy that case III indeed indicates another force transmission pathway that flow shear stress is directly transmitted via the core protein, especially in the situation with a bulk of sugar chains removed, and the alternative force transmission pathway was discussed in our previous studies $^{25,30}$.

\section{Mechanotransduction and microvascular permeability}

The EG is essential for regulating the microvascular permeability and for maintaining the transduction of the mechanical signals from the blood flow. However, when HS sugar chains are disrupted, contrasting influences on mechanotransduction and microvascular barrier are manifested: the mechanotransduction is impaired ${ }^{47}$ whereas the microvascular 
permeability is affected marginally ${ }^{5,48}$. Rehm et al. proposed a two-barrier hypothesis that the EG is regarded as an additional component to the barrier formed by the endothelial cells 48. In this hypothesis, significant alteration in permeability occurs only when both the EG and the endothelial cells are disturbed. The disruption of EG without alteration of endothelial cells only results in a short-time permeability changes and the changes will vanish eventually. Such hypothesis can successfully explain for the independence of permeability on the disruption of HS sugar chains. However, how the hypothesis can be linked to the mechanotransduction of EG is unclear and requires further exploration.

In the following discussion, we present a hypothesis which relates the role of EG in mechanotransduction to its function as a microvascular barrier. In this hypothesis, the EG regulates the mechanotransduction by altering the median value and variation range of the scissor angle (The median value modulates the deformation of cells and the variation range determines the intensity of force disturbing the cytoskeleton.); the EG governs the microvascular barrier via controlling the scissor angle which will affect the intercellular cleft, an important pathway for vascular permeability ${ }^{49}$. In the vascular homeostasis, the Syn- 4 core proteins are connected to the actin cortical web ${ }^{36}$ in the cytoplasm of endothelial cells, and the intercellular clefts between the endothelial cells regulate the microvascular transport, as illustrated in Figure 5A. When the EG is disrupted (for example sugar chains shed from the core proteins), a reduction in the sugar chain number by $17 \%$ (from 18 in case I to 15 in case II, Figure 4C) can affect the mechanotransduction by $55 \%$; however, the same disruption in sugar chains can only lead to a small scissor angle change by about $3 \%$. The pertinent small 
deformation of the cytoskeleton is inadequate to cause the cleft dimensional changes, leaving the microvascular permeability unchanged, as displayed in Figure 5B. Finally, a new homeostasis after the removal of the sugar chains is reached as shown in Figure 5C. Please note that stress fibres and junction network, such as tight junction and adherens junction, will also be altered when the EG is disrupted ${ }^{36}$. The alterations in the stress fibres and junctions are beyond the scope of this research and are not illustrated in Figure 5.

Our findings open a new road to revisit classic topics in understanding the functionality of EG in mechanotransduction and reconcile longstanding debates about the force transmission threshold which previous theories are inadequate to resolve. The present research also provides a new hypothesis which, for the first time, relates the mechanotransduction to the microvascular barrier of the EG. Such a hypothesis explains for the contrasting influences of the shedding of sugar chains on the EG functions of mechanotransdcution and microvascular barrier. In the future research, a comprehensive EG model containing other core protein types (e.g. glypicans and perlecans) is expected to reveal additional details about mechanosensing and mechanotransduction.

\section{Methods}

\section{Molecular Dynamics Modelling}

MD simulations can provide the ultimate detail concerning temporal trajectories of individual atoms. Thus, they can be used to address specific questions about the properties of a model system, often more easily than experiments on the actual system ${ }^{50}$. The basic idea 
behind an MD simulation is to calculate the force exerted on each atom by all other atoms using Newton's laws of motion and to update position and velocity of each atom using the calculation results in each timestep. The resulting trajectories of atoms describe the atomic-level configuration of the system at every point during the simulated time interval ${ }^{51}$. The forces in an MD simulation are calculated using molecular mechanics force fields, which are typically obtained from quantum mechanical calculations or fitting to experimental data. For more details, the readers are referred to Ref. ${ }^{52}$. To implement a flow simulation, one effective strategy is to impose forcing scheme on water molecules as practiced in previous studies 5326 .

\section{Flow/EG model}

The main building block of the model, one EG element is composed of a core protein and six sugar chains. Syn-4 proteoglycan and heparin sulphate (HS) sugar residues are selected to model the EG core protein and sugar chains, respectively. Figure 1 illustrates the flow/EG system used in the present study. As shown in Figure 1A, the whole space is divided into two compartments by the lipid bilayer. Above the lipid bilayer is the ectodomain, representing the space outside the endothelial cells, where flow passes by. This region contains negatively-charged HS sugar chains, Syn-4 ectodomain in connection with HS sugar chains, water molecules and ions. Below the lipid bilayer is the cytoplasm, representing the inner space of the cell, which is filled with the Syn-4 cytoplasmic protein, water molecules and ions ${ }^{25}$. Syn-4 transmembrane dimer with secondary structure of $\alpha$-helix constitutes the transmembrane region of the EG (Figure 1B). All the biomolecules are solvated in the $\mathrm{NaCl}$ 
solution with a concentration of $0.1 \mathrm{M}$. The simulation box is a hexagonal prism with an area of $820 \mathrm{~nm}^{2}$ and height of $72 \mathrm{~nm}$. The entire flow/EG system comprises $~ 5,800,000$ atoms in total.

\section{Protocol Details}

The TIP3P water model ${ }^{54}$ is adopted to simulate water molecules. The CHARMM biomolecular force field ${ }^{55}$ is applied on proteins and the lipid bilayer. Force field parameters for sugar chains and graphene layers are from previous studies ${ }^{24}$.

Before conducting the production flow simulations, the system was equilibrated under isothermal-isobaric (NPT) and canonical (NVT) ensembles. In the flow simulations, the velocity Verlet integration method ${ }^{56}$ was used to advance the positions and velocities of the atoms in time. A 2-fs time step, and PME electrostatics with a grid density of $1 \AA^{-3}$ are used. The SETTLE algorithm ${ }^{57}$ was used to enable the rigid bonds connected to all hydrogen atoms. The van der Waals interactions were calculated using a cut-off of $12 \AA$ with a switching function starting at $10 \AA^{24}$.

All MD simulations were performed using the software suite NAMD $2.9^{58}$. The visualisation of the molecular structures is performed via the VMD ${ }^{59}$ package. All parallel simulations and non-visualised post-processing are conducted on ARCHER, UK's national supercomputing service.

This article is protected by copyright. All rights reserved. 
Details about the construction of the flow/EG system and the protocol information can be found in Ref. ${ }^{25,27,28}$

\section{Flow simulations and case set-up}

To mimic flow where appropriate, external forces were imposed on the water oxygens in the ectodomain. In a basic flow case, an external force of $0.003 \mathrm{fN}$ was selected, a value that produces an average flow velocity in a physiologically reasonable range ${ }^{25}$. The physical time of the basic case is $30 \mathrm{~ns}$. By varying the external force to $0.001 \mathrm{fN}$ and $0.002 \mathrm{fN}$, two additional flow situations were simulated with a physical time of 15 ns. A control case presenting a stationary situation without flow was also mimicked, and the external force was set to 0 with a physical time of $8 \mathrm{~ns}$.

\section{Statistical Information}

The sample sizes of the $0.003 \mathrm{fN}$-flow, $0.002 \mathrm{fN}$-flow, $0.001 \mathrm{fN}$-flow and no-flow cases are 150, 105, 105 and 40, respectively. The sample sizes for both cases with shedding sugar chains are 105

\section{Acknowledgements}

The authors greatly appreciate helpful discussions and valuable input from Prof. Haipeng Gong of Tsinghua University.

This article is protected by copyright. All rights reserved. 
This work was supported by the UK Engineering and Physical Sciences Research Council under the project UK Consortium on Mesoscale Engineering Sciences (UKCOMES) (Grants No. EP/L00030X/1 and No. EP/R029598/1).

\section{Conflict of interest}

The authors declare that they have no competing interests.

\section{References}

1. Curry FRE, Adamson RH. Vascular permeability modulation at the cell, microvessel, or whole organ level: towards closing gaps in our knowledge. Cardiovascular Research. 2010;87(2):218-229.

2. Reitsma S, Slaaf DW, Vink H, van Zandvoort MAMJ, oude Egbrink MGA. The endothelial glycocalyx: composition, functions, and visualization. Pflügers Archiv European Journal of Physiology. 2007;454(3):345-359.

3. Rabelink TJ, de Zeeuw D. The glycocalyx - linking albuminuria with renal and cardiovascular disease. Nature Reviews Nephrology. 2015;11:667.

4. Sieve I, Munster-Kuhnel AK, Hilfiker-Kleiner D. Regulation and function of endothelial glycocalyx layer in vascular diseases. Vascul Pharmacol. 2018;100:26-33.

5. Henderson-Toth CE, Jahnsen ED, Jamarani R, Al-Roubaie S, Jones EA. The glycocalyx is present as soon as blood flow is initiated and is required for normal vascular development. Developmental biology. 2012;369(2):330-339.

6. Liu J, Kang H, Ma X, et al. Vascular Cell Glycocalyx-Mediated Vascular Remodeling 
Induced by Hemodynamic Environmental Alteration. Hypertension. 2018;71(6):1201-1209.

7. Tarbell JM, Cancel LM. The glycocalyx and its significance in human medicine. $J$ Intern Med. 2016;280(1):97-113.

8. Dane MJC, Berg BMvd, Lee DH, et al. A microscopic view on the renal endothelial glycocalyx. American Journal of Physiology-Renal Physiology. 2015;308(9):F956-F966.

9. Song JW, Zullo JA, Liveris D, Dragovich M, Zhang XF, Goligorsky MS. Therapeutic Restoration of Endothelial Glycocalyx in Sepsis. The Journal of pharmacology and experimental therapeutics. 2017;361(1):115-121.

10. Qazi H, Palomino R, Shi ZD, Munn LL, Tarbell JM. Cancer cell glycocalyx mediates mechanotransduction and flow-regulated invasion. Integrative biology : quantitative biosciences from nano to macro. 2013;5(11):1334-1343.

11. Wang N, Tytell JD, Ingber DE. Mechanotransduction at a distance: mechanically coupling the extracellular matrix with the nucleus. Nat Rev Mol Cell Bio. 2009;10(1):75-82.

12. Polacheck WJ, German AE, Mammoto A, Ingber DE, Kamm RD. Mechanotransduction of fluid stresses governs 3D cell migration. Proc Natl Acad Sci U S A. 2014;111(7):2447-2452.

13. Haining AWM, Rahikainen R, Cortes E, et al. Mechanotransduction in talin through the interaction of the R8 domain with DLC1. PLOS Biology. 2018;16(7):e2005599.

14. Turner MS, Sens P. Gating-by-tilt of mechanically sensitive membrane channels. Phys 
Rev Lett. 2004;93(11):118103.

15. Sabass B, Stone HA. Role of the Membrane for Mechanosensing by Tethered Channels. Phys Rev Lett. 2016;116(25):258101.

16. Uroz M, Wistorf S, Serra-Picamal X, et al. Regulation of cell cycle progression by cell-cell and cell-matrix forces. Nature Cell Biology. 2018;20(6):646-654.

17. Tzima E, Irani-Tehrani $\mathrm{M}$, Kiosses $\mathrm{WB}$, et al. A mechanosensory complex that mediates the endothelial cell response to fluid shear stress. Nature (London). 2005;437(7057):426-431.

18. Chatterjee S. Endothelial Mechanotransduction, Redox Signaling and the Regulation of Vascular Inflammatory Pathways. Frontiers in Physiology. 2018;9(524).

19. Dogné S, Flamion B, Caron N. Endothelial Glycocalyx as a Shield Against Diabetic Vascular Complications. Arteriosclerosis, Thrombosis, and Vascular Biology. 2018;38(7):1427-1439.

20. Florian JA, Kosky JR, Ainslie K, Pang Z, Dull RO, Tarbell JM. Heparan sulfate proteoglycan is a mechanosensor on endothelial cells. Circ Res. 2003;93(10):e136-142.

21. Tarbell JM, Simon SI, Curry FRE. Mechanosensing at the Vascular Interface. Annual Review of Biomedical Engineering, Vol 16. 2014;16:505-532.

22. Dabagh M, Jalali P, Butler PJ, Randles A, Tarbell JM. Mechanotransmission in endothelial cells subjected to oscillatory and multi-directional shear flow. Journal of the Royal Society, Interface. 2017;14(130).

23. Eriksson M, Lindhorst TK, Hartke B. Differential effects of oligosaccharides on the 
hydration of simple cations. J Chem Phys. 2008;128(10):105105.

24. Cruz-Chu ER, Malafeev A, Pajarskas T, Pivkin IV, Koumoutsakos P. Structure and response to flow of the glycocalyx layer. Biophys J. 2014;106(1):232-243.

25. Jiang XZ, Gong H, Luo KH, Ventikos Y. Large-scale molecular dynamics simulation of coupled dynamics of flow and glycocalyx: towards understanding atomic events on an endothelial cell surface. Journal of The Royal Society Interface. 2017;14(137).

26. Pikoula M, Tessier MB, Woods RJ, Ventikos Y. Oligosaccharide model of the vascular endothelial glycocalyx in physiological flow. Microfluidics and Nanofluidics. 2018;22(2):21.

27. Jiang XZ, Feng M, Ventikos Y, Luo KH. Regimes of Flow over Complex Structures of Endothelial Glycocalyx: A Molecular Dynamics Simulation Study. Scientific Reports. 2018;8(1):5732.

28. Jiang XZ, Feng M, Luo KH, Ventikos Y. Large-scale molecular dynamics simulation of flow under complex structure of endothelial glycocalyx. Computers \& Fluids. 2018;173:140-146.

29. Jiang XZ, Luo KH, Ventikos Y. Reducing Salt Intake and Exercising Regularly: Implications From Molecular Dynamics Simulations of Endothelial Glycocalyx. Frontiers in Physiology. 2018;9(1667).

30. Jiang XZ, Lu Y, Luo KH, Ventikos Y. Understanding endothelial glycocalyx function under flow shear stress from a molecular perspective. Biorheology. 2019.

31. Jiang XZ, Ventikos Y, Luo KH. Microvascular ion transport through endothelial glycocalyx layer: new mechanism and improved Starling principle. American Journal 
of Physiology-Heart and Circulatory Physiology. 2019;317(1):H104-H113.

32. Elfenbein A, Simons M. Syndecan-4 signaling at a glance. Journal of Cell Science. 2013;126(17):3799-3804.

33. Baeyens N, Mulligan-Kehoe MJ, Corti F, et al. Syndecan 4 is required for endothelial alignment in flow and atheroprotective signaling. Proceedings of the National Academy of Sciences. 2014;111(48):17308-17313.

34. Reitsma S, Slaaf DW, Vink H, van Zandvoort MAMJ, oude Egbrink MGA. The endothelial glycocalyx: composition, functions, and visualization. Pflugers Arch. 2007;454(3):345-359.

35. Weinbaum S, Zhang X, Han Y, Vink H, Cowin SC. Mechanotransduction and flow across the endothelial glycocalyx. Proc Natl Acad Sci $U$ S A. 2003;100(13):7988-7995.

36. Thi MM, Tarbell JM, Weinbaum S, Spray DC. The role of the glycocalyx in reorganization of the actin cytoskeleton under fluid shear stress: a "bumper-car" model. Proceedings of the National Academy of Sciences of the United States of America. 2004;101(47):16483-16488.

37. Bakan A, Meireles LM, Bahar I. ProDy: protein dynamics inferred from theory and experiments. Bioinformatics (Oxford, England). 2011;27(11):1575-1577.

38. Weinbaum S, Tarbell JM, Damiano ER. The Structure and Function of the Endothelial Glycocalyx Layer. Annual Review of Biomedical Engineering. 2007;9(1):121-167.

39. Binnig G, Quate CF, Gerber C. Atomic Force Microscope. Physical Review Letters. 1986;56(9):930-933.

This article is protected by copyright. All rights reserved. 
40. Giessibl FJ. Advances in atomic force microscopy. Reviews of Modern Physics. 2003;75(3):949-983.

41. Steffen P, Verdier C, Wagner C. Quantification of depletion-induced adhesion of red blood cells. Phys Rev Lett. 2013;110(1):018102.

42. Staple DB, Payne SH, Reddin ALC, Kreuzer HJ. Model for Stretching and Unfolding the Giant Multidomain Muscle Protein Using Single-Molecule Force Spectroscopy. Phys Rev Lett. 2008;101(24).

43. Kamm RD, Kaazempur-Mofrad MR. On the molecular basis for mechanotransduction. Mech Chem Biosyst. 2004;1(3):201-209.

44. Tarbell JM, Weinbaum S, Kamm RD. Cellular fluid mechanics and mechanotransduction. Ann Biomed Eng. 2005;33(12):1719-1723.

45. Oberhauser AF, Hansma PK, Carrion-Vazquez M, Fernandez JM. Stepwise unfolding of titin under force-clamp atomic force microscopy. Proc Natl Acad Sci U S A. 2001;98(2):468-472.

46. Cruickshank K, Riste L, Anderson SG, Wright JS, Dunn G, Gosling RG. Aortic pulse-wave velocity and its relationship to mortality in diabetes and glucose intoleranc: an integrated index of vascular function? Circulation. 2002;106(16):2085-2090.

47. Moon JJ, Matsumoto M, Patel S, Lee L, Guan JL, Li S. Role of cell surface heparan sulfate proteoglycans in endothelial cell migration and mechanotransduction. Journal of cellular physiology. 2005;203(1):166-176.

48. Rehm M, Zahler S, Lotsch M, et al. Endothelial glycocalyx as an additional barrier 
determining extravasation of $6 \%$ hydroxyethyl starch or $5 \%$ albumin solutions in the coronary vascular bed. Anesthesiology. 2004;100(5):1211-1223.

49. Tarbell JM. Shear stress and the endothelial transport barrier. Cardiovasc Res. 2010;87(2):320-330.

50. Karplus M, McCammon JA. Molecular dynamics simulations of biomolecules. Nature Structural Biology. 2002;9:646.

51. Hollingsworth SA, Dror RO. Molecular Dynamics Simulation for All. Neuron. 2018;99(6):1129-1143.

52. González M. Force fields and molecular dynamics simulations. École thématique de la Société Française de la Neutronique. 2011;12:169-200.

53. Kotsalis EM, Walther JH, Koumoutsakos P. Multiphase water flow inside carbon nanotubes. International Journal of Multiphase Flow. 2004;30(7):995-1010.

54. Jorgensen WL, Chandrasekhar J, Madura JD, Impey RW, Klein ML. Comparison of simple potential functions for simulating liquid water. $J$ Chem Phys. 1983;79(2):926-935.

55. MacKerell AD, Bashford D, Bellott M, et al. All-atom empirical potential for molecular modeling and dynamics studies of proteins. $J$ Phys Chem B. 1998;102(18):3586-3616.

56. Allen MPA, Tildesley DJ. Computer Simulation of Liquids. New York: Oxford University Press; 1987.

57. Miyamoto S, Kollman PA. Settle - an Analytical Version of the Shake and Rattle Algorithm for Rigid Water Models. Journal of Computational Chemistry. 
1992;13(8):952-962.

58. Phillips JC, Braun R, Wang W, et al. Scalable molecular dynamics with NAMD. Journal Computat Chem. 2005;26(16):1781-1802.

59. Humphrey W, Dalke A, Schulten K. VMD: Visual molecular dynamics. J Mol Graphics Modell. 1996;14(1):33-38.

\section{Legends to figures}

Figure 1 An all-atom model of the flow/EG system. A. The structure of the EG element published in Ref. ${ }^{24}$ with Syndecan-4 and HS chains is adopted in the construction of the flow/EG system. Three EG elements are embedded in a lipid bilayer amid water molecules. The entire system contains $\sim 5,800,000$ atoms. Water molecules are partly shown, and ions are not shown. Flow is in the $x$ direction. B. Secondary structure of the EG core protein. The transmembrane Syn-4 protein is a helix dimer, and Syn-4 ectodomain and transmembrane part are linked by flexible coils. EA1, EA2, EB1 and EB2 are four subdomains of the Syn-4 ectodomain part. TA1 is the first coil of Chain A from the Syn-4 transmembrane dimer with TB1 the first coil of Chain B. TA2 and TB2 are the middle coils of the transmembrane parts of Chain A and Chain B, respectively. TA3 and TB3 individually represent the tail coils of the transmembrane dimer. $\mathrm{COM}_{\mathrm{T}}$ is the centre of mass of the Syn-4 transmembrane dimer.

This article is protected by copyright. All rights reserved. 
Figure 2 Dynamics of the EG core protein subdomains under flow and stationary conditions. A. Unsynchronised angle changes between (i) the basic flow case (with external force of $0.003 \mathrm{fN}$ ) and (ii) the control case (stationary condition) suggest the soft-matter features of the Syn- 4 core protein. Normalized time is ratio of the time of interest to the total time simulated. $\theta_{\mathrm{T}}$ is to measure the relative position of the transmembrane part to $\mathrm{COM}_{\mathrm{T}}$, and $\theta_{\mathrm{E} 1}$ and $\theta_{\mathrm{E} 2}$ represent the relative positions of the subdomains of Syn- 4 ectodomain to $\mathrm{COM}_{\mathrm{T}}$. B. Unsynchronised changes in distances between Syn-4 subdomains under flow and stationary situations. C. Helix lengths of the Syn-4 dimer under flow and stationary situations. The insignificant changes in helix lengths suggest inextensible transmembrane Syn-4 dimer. $d_{\mathrm{TA}}$ and $d_{\mathrm{TB}}$ are the helix lengths of the transmembrane parts of Chain $\mathrm{A}$ and Chain $\mathrm{B}$, respectively.

\section{Figure 3 Force transmission mode via the transmembrane part of the core protein and}

its order of magnitude. A. $\theta_{\mathrm{AB}}$ is to quantify the relevant position of the two chains of the dimer, and large angle variations of $\theta_{\mathrm{AB}}$ can be found in the flow group $(p<0.001$ by Fligner-Killeen Test). B. Probability density distributions of $\theta_{\mathrm{A}}$ (to measure the bending of the transmembrane $\alpha$-helix of Chain A), $\theta_{\mathrm{B}}$ (to measure the bending of the transmembrane $\alpha$-helix of Chain B) and $\theta_{\mathrm{AB}}$. Flow closes the angle of $\theta_{\mathrm{AB}}$ by 3.1 degrees, and $\theta_{\mathrm{A}}$ indicating the stretching of Chain A increases by about 3 degrees. C. PCA analysis of the motions of transmembrane protein. Scissor motion of the two chains is the first principle motion mode of the transmembrane protein, followed by two bending modes of the dimer in two planes. (Animations for the three motion modes can be found in Supplementary Movies 1-3, 
respectively.) D. PDF for forces to mimic the closure of the dimer in SMD simulations. A constant velocity of $0.0018 \mathrm{~m} \cdot \mathrm{s}^{-1}$ is imposed on the end of one chain of the transmembrane dimer. To maintain the constant pulling velocity, forces with an order of magnitude of 10 100 $\mathrm{pN}$ are required.

Figure 4 Changes in flow conditions and sugar chain numbers modify the scissor motion of the transmembrane Syn-4 dimer. A. $\theta_{\mathrm{AB}}$ changes under varying flow conditions in terms of external force. B. Initial configurations of the shedding scenarios applied in this research (top views only with sugar chains). $\mathrm{N}$ is the number of sugar chains. The intact case (case I) contains 18 sugar chains; by removing the sugar chains marked in red in Case I, 15 sugar chains are retained in case II; and by removing the sugar chains marked in blue in Case II, 9 sugar chains are retained in case III. C. $\theta_{\mathrm{AB}}$ changes under the shedding sugar chain scenarios. $(\dagger p<0.01 ; \ddagger p<0.001$ by ANOVA).

Figure 5 The proposed hypothesis to explain the contrasting influences from disruption of HS sugar chains in mechanotransduction and microvascular permeability.

A. In the vascular homeostasis, the EG core proteins are connected to actin cortical web in the cytoplasma of endothelial cells, and intercellular clefts are the main pathways for microvascular transport. B. The removal of sugar chains which significantly affects mechanotransduction only causes small deformations of the endothelial cells. The small deformation is inadequate to vary the microvascular permeability. C. New homeostasis is reached after the disruption of EG.

This article is protected by copyright. All rights reserved. 

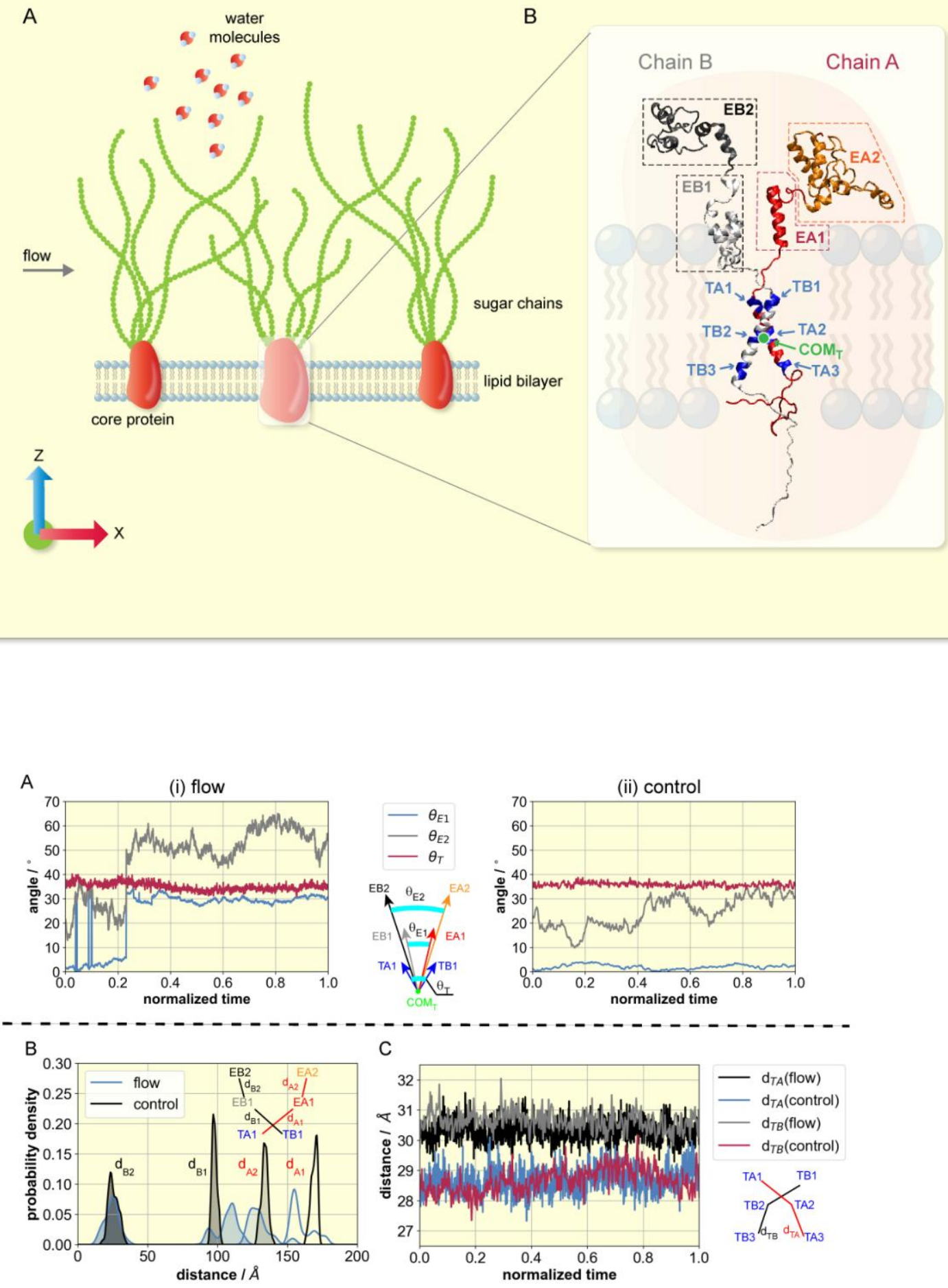

This article is protected by copyright. All rights reserved. 

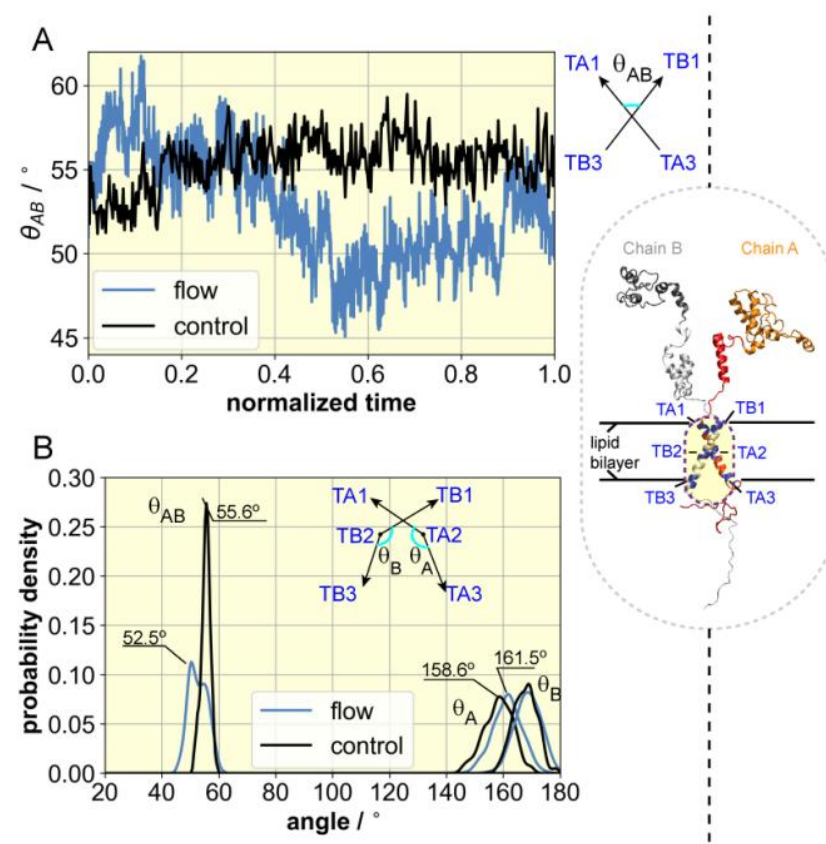
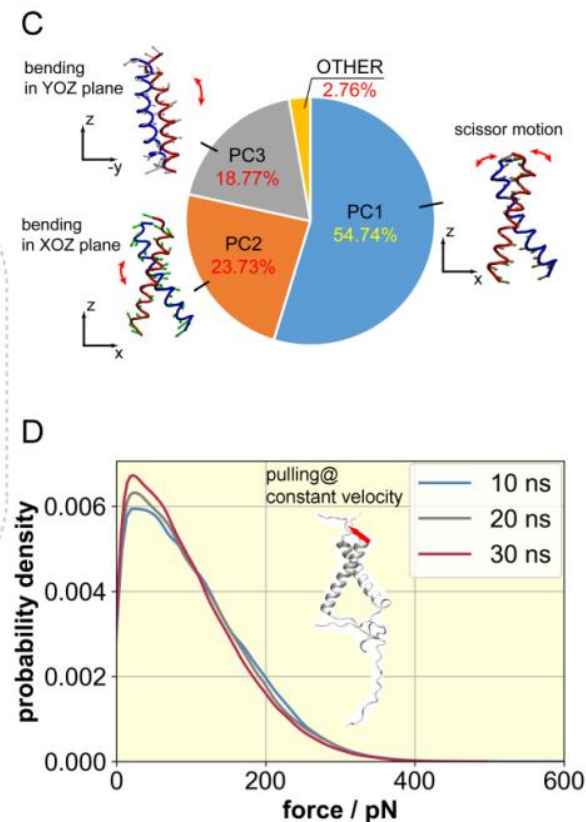

This article is protected by copyright. All rights reserved. 


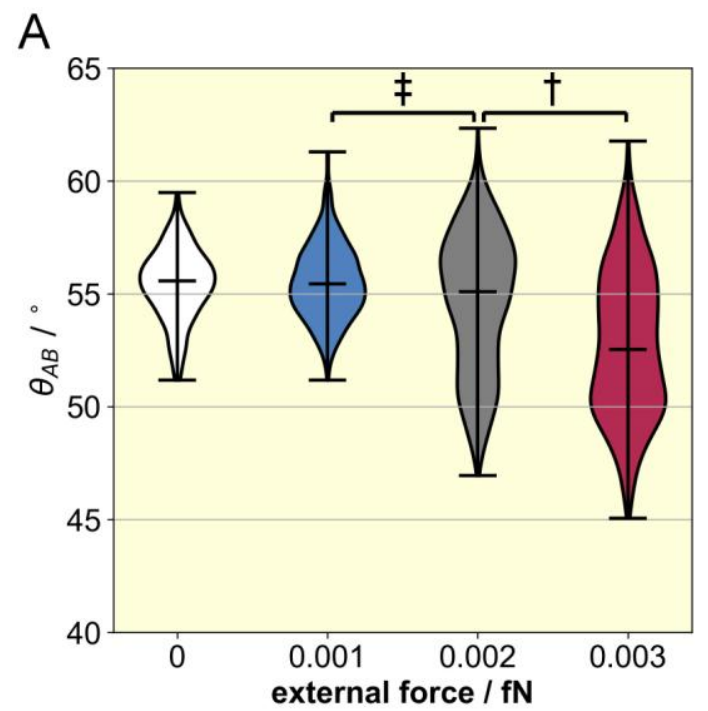

B
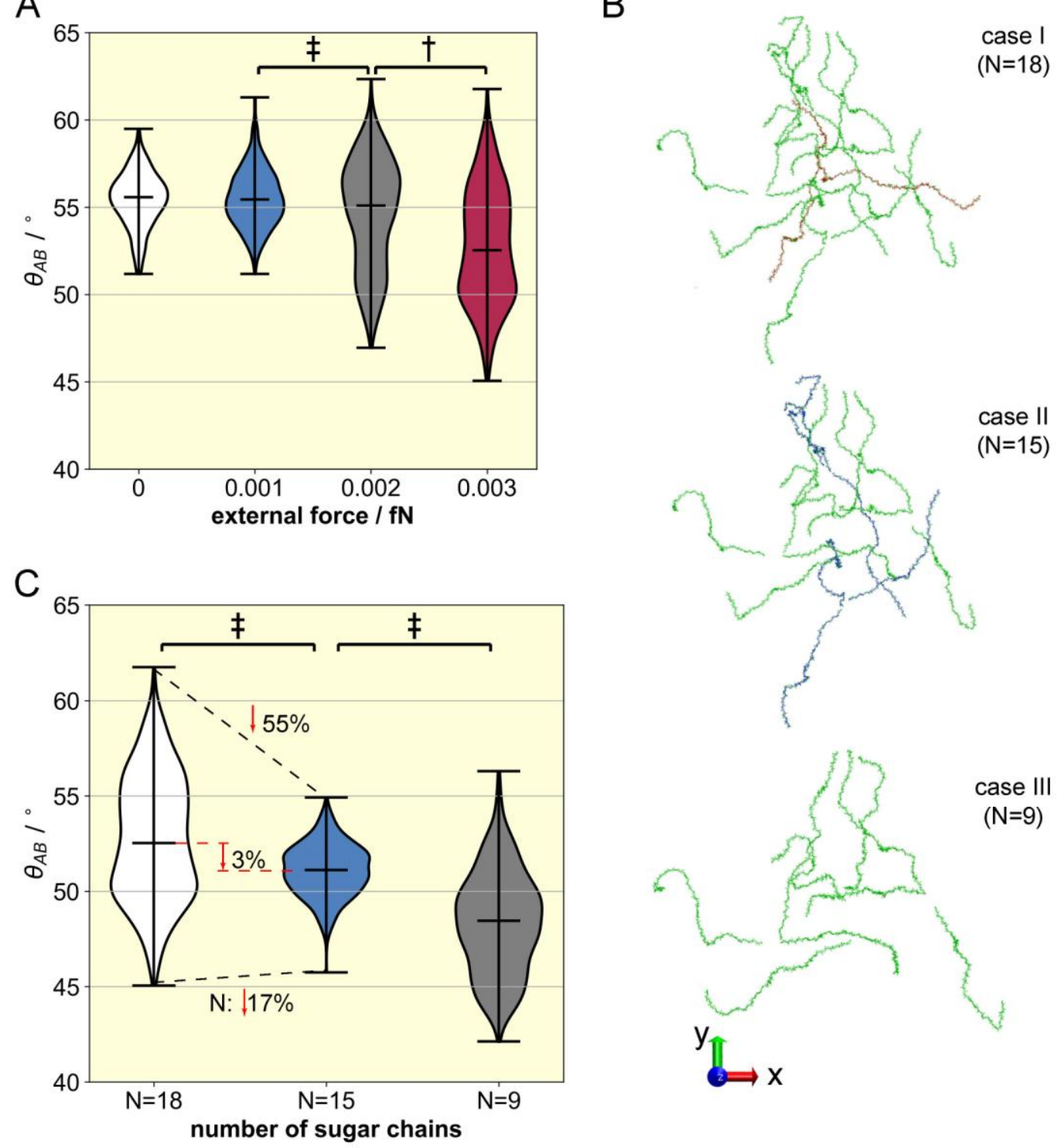

$(\mathrm{N}=9)$

This article is protected by copyright. All rights reserved. 
A

homeostatis

(intact EG)

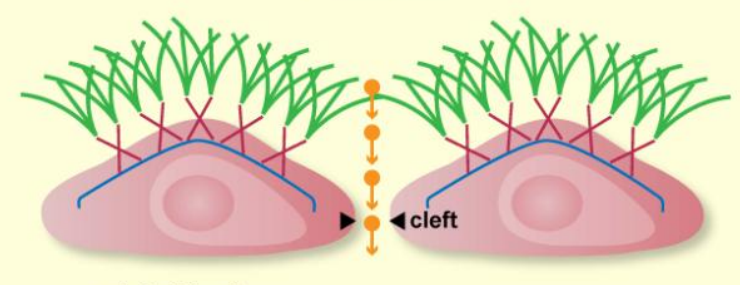

endothelial cells

C homeostatis

(removal of sugar chains)

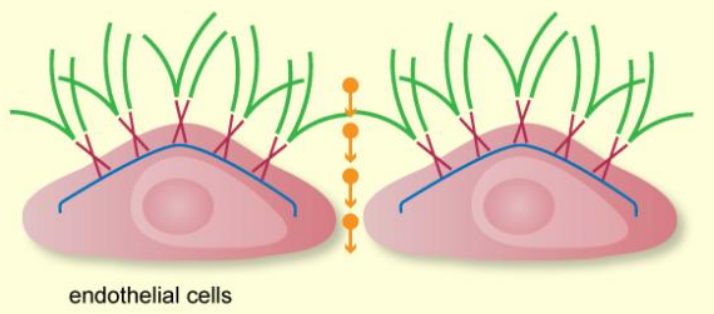

B

disturbance (removal of sugar chains)

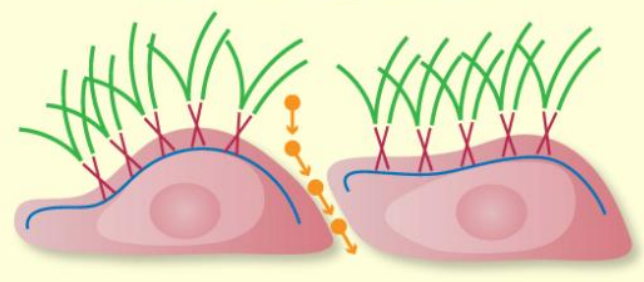

endothelial cells

$$
\begin{aligned}
& \text { EG core } \\
& \text { protein }
\end{aligned} \quad \begin{aligned}
& \text { biomulecules/ } \\
& \text { particles/... }
\end{aligned}
$$

This article is protected by copyright. All rights reserved. 